\title{
ANALYSIS OF PERIOD CHANGES OF YY ERIDANI
}

\author{
CHUN-HWEY KIM \\ Institute of Space Science and Astronomy \\ 36-1 Whaamdong, Yuseonggu \\ Daejeon 305-348 \\ Korea
}

\begin{abstract}
Four times of minimum lights from six nights photoelectric observations of YY Eri during the observing seasons from 1983 to 1984 are derived. With these and previously published photoelectric minima, new light elements are determined. A detailed numerical investigation of times of minima shows that the orbital period has changed sinusoidally with a period of about 24 years. These changes of period are investigated in terms of two plausible causes : a light-time effect due to an unseen third body and asymmetries of light curves due to stellar activities of one component or both.
\end{abstract}

\section{INTRODUCTION}

The eclipsing binary YY Eri(BD-100858, $\left.P=0^{d} .321, S_{p}=G 5\right)$ is a W UMa type star. The light and the orbital period have been reported to be variable(Huruhata et al. 1953, Kwee 1958, Purgathofer and Purgathofer 1960, Yamasaki 1975, Strauss 1976, Budding 1983).

As to the behavior of the orbital period Kwee found it has increased since the eariler observations and this fact was confirmed by the Purgathofers who suggested a quasi-parabolic variation. Also, Strauss noticed the non-linear changes in the period and Budding remarked that such variation might be linked up with the intrinsic variation of the system itself.

Up to date no clear picture for the period behavior of YY Eri were made. In this regards we made photoelectric observations of this intriguing star and analyzed all the photoelectric minima.

\section{OBSERVATIONS and O-C DIAGRAM}

YY Eri was observed on six nights from December 1983 to December 1984 with a $61 \mathrm{~cm}$ reflector at the Sobaeksan Astronomical Observatory. The photometer is equipped with a refrigerated 1P21 photomultiplier and an UBV Johnson photometric system. A total of 343 observations for YY Eri were secured in three colors. From our observations four minimum times were derived with the method of Kwee and van Woerden(1956, hierafter $\mathrm{KW})$.

In our period analysis with all the collected 35 photoelectric times of minimum 
we found that the orbital period of YY Eri can be represented in a non-1inear light elements with a sine function. Thus the minima are fitted to a light elements with a sine function by the method of iterative differential corrections. The final solution is :

$$
\begin{aligned}
& \text { Min I }=\text { JD } 2433617.5228+0^{d} .32149591 E-0^{d} .00336 \sin \left(0^{\circ} .01307 E+50^{\circ} .7\right) \\
& \pm 2 \quad \pm 1 \quad \pm 26 \quad \pm 17 \quad \pm 4.2
\end{aligned}
$$

This equation may be judged from Figure 1 where we have plotted the $0-C$ residuals for the photoelectric minima with respect to the linear parts of equation (1).

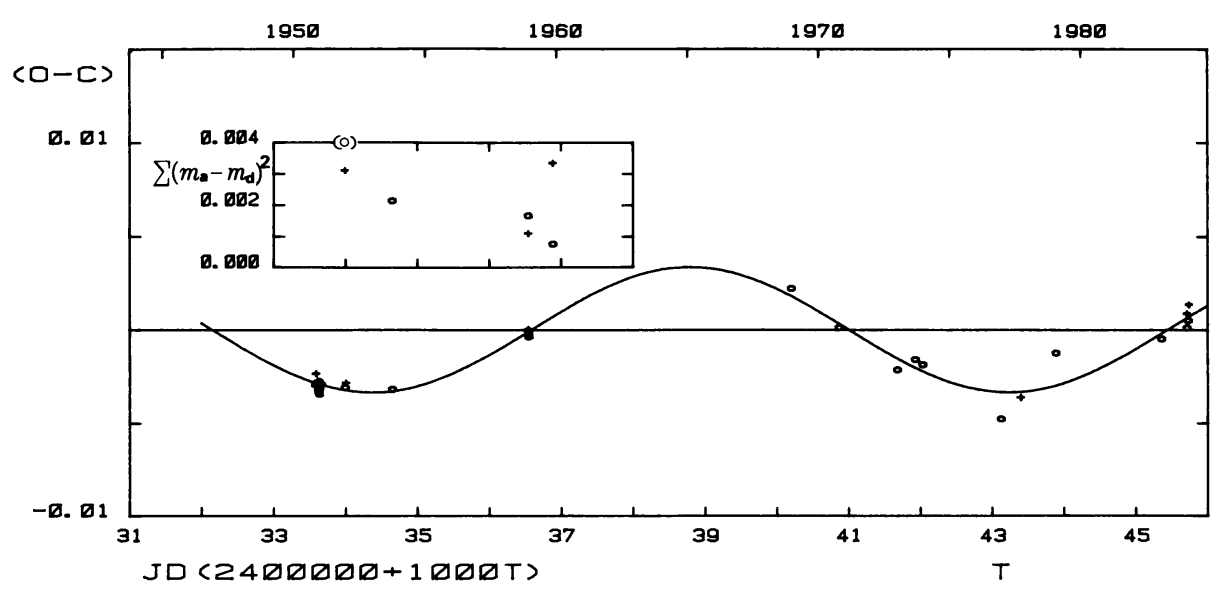

Figure 1. O-C diagram of YY Eri. Open circles and plus signs denote primary and secondary minima, respectively. Continuous lines represent the non- 1 inear term of formula (1). Variations of asymmetries are plotted in the upper box (o : yellow, + : blue, see text).

\section{THIRD BODY INTERPRETATION}

As the behaviors of secondary minima of YY Eri follow those of primaries we propose an unseen third body in YY Eri system. From the amplitude and the angular speed per binary period of sine term of the formula (1) the corresponding period and mass function are calculated as $P^{\prime}=24.24$ years and $f(M)=0.000333 M_{e}$, respectively. The masses of the third body have, ranges of $0.14 \mathrm{M}_{\mathrm{\theta}} \sim 0.38 \mathrm{M}$, for the inclination ranges of $90^{\circ} \sim 20^{\circ}$. In this calcalution $2.12 M_{0}$ of the ecliping pair(Nesci et al. 1986) is used.

The derived masses are quite small and hardly detectable as a third body spectrum. YY Eri is $50 \sim 70$ pc distant from our Sun(Dworak 1973, Rucinski 1983). And the semimajor axis(a sin $i)$ of the orbit of the third body about the center of mass of the close pair is $0.581 \mathrm{AU}$. Its corresponding angular size at a distance of $50 \mathrm{pc}$ would be 0 ." $01 \sim 0$ ". 03 for $i=90^{\circ} \sim 10^{\circ}$, and for $70 \mathrm{pc}, 0$. 008 $\sim 0$. 023 . Relative astrometric observations at resolutions substantially better than these may be needed to prove whether the suggested third-body exists or not. 


\section{ASYMMETRIES OF ECLIPSE LIGHT CURVES}

Many attempts to relate period changes to variations in light curves for well observed systems, for example, such as VW Cep, have been made (Kwee 1966, Yamasaki 1982. Karimie 1983). Variable asymmetric light curves could result in the variable phase shifts of minimum times which mimic dynamical changes of period (Kwee 1966). In this line it is investigated whether the similar situation for period variations of YY Eri is possible, because the amplitude of about 0.003 of period changes is quite small compared with those of other W UMa-type binaries(Yamasaki 1975). In the 0-C diagram of Figure 1 light curves observed near top or bottom of the sine curve may be expected to be more asymmetrical than those near nodal points. Unfortunately the observations of YY Eri so far are not sufficient to test correlations between changes of period and light curves quantitatively.

The individual observations have been published by Huruhata et al. (1953), Kwee(1958) and Purgathofer and Purgathofer(1960). Kwee's observations wi thout filters were made only during primary eclipse. Among them the observations of Huruhata et al. and Kwee are near the bottom of the sine curve (JD2433989 - JD2434648). The Purgathofers' and ours are near nodal positions (JD2436540 JD2436544, JD2445709 JD2445741) separated from the time interval of about 9200 days. In Figure 2 each eclipse light curve in yellow and blue bandpasses is replotted with the linear terms of equation (1). Continuous lines are polynomial curves best fitted to observations within the same phase interval of $0.92 \sim 1.08$. Different degrees of polynomials for each light curves are used because each light curve has different sets of data in number and shape. Dotted lines are reflections of descending polynomial curves with axes of minimum epochs(drawn as arrows in Figure 2) determined wi th the KW method.

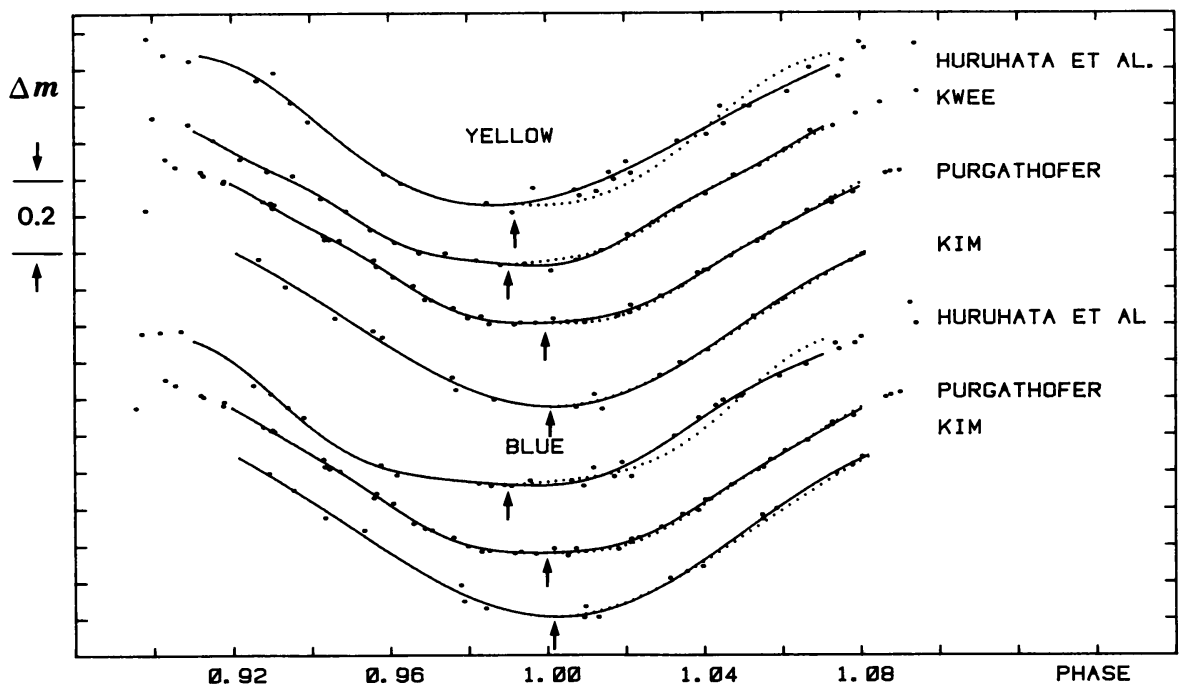

Figure 2. Variations of eclipse light curves of YY Eri. Continuous lines are polynomial curves and dotted ones are reflections of descending polynomial curves. Arrows denote the epochs determined by the KW method. 
Amounts of asymmetries of each light curves are calculated in terms of magnitude differences $\left(\Sigma\left(m_{a}-m_{d}\right)^{2}\right)$ between the descending and ascending curves. These are plotted in the upper part of $0-C$ diagram of Figure 1 . Our observations are plotted at the corresponding phase by shifting backward one cycle of the sine curve. As seen in the figure, there seems to be some changes of asymmetries in eclipse light curves. But it is uncertain whether the variations are periodic or not because of lack of observations.

\section{CONCLUSION}

Among two hypotheses suggested for changes of period of YY Eri the light time effect due to a third body seems to be, at present, preferable to the shifts of minimum epochs due to asymmetries of eclipse light curves. Various observations (photometry, astrometry, and spectroscopy) are needed.

ACKNOWLEDGEMENT. This work was supported financially in part by MOST grant B9114 in 1991, for which CHK expresses his gratitude.

\section{REFERENCES}

Budding, E. 1983, IBVS, 2300

Dworak, T. Z. 1973, IBVS, 846

Huruhata, M. , Dambara, T. and Kitamura, M. 1953, Tokyo Ann. Second Ser., 3, 227

Karmie, M. T. 1983, Ap\&SS, 92, 53

Kwee, K. K. 1958, BAN, 14, 131

Kwee, K. K. 1966, BAN, 18, 448

Kwee, K. K. and van Woerden, H. 1956, BAN, 12,327

Nesci, R., Maceroni, C., Milano, L. and Russo, G. 1986, A\&A, 159, 142

Purgathofer, A. and Purgathofer, I. 1960, Mitt. Wien, 10, 195

Rucinski, S. M. 1983, A\&A, 127, 84

Strauss, F. M. 1976, PASP, 88, 531

Yamasaki, A. 1975, Ap\&SS, 34, 413

Yamasaki, A. 1982, Ap\&SS, 85, 43 